the theorems employed in analysis which rest on intuition and analogy. Thus no mention has been made of the theorem required to justify the ordinary use of the inner normal. But the theorems we have stated are sufficient to show in what direction further proof is necessary if the fundamental theorems of analysis are really to rest on an arithmetic basis.

HARVARD UNTVERSTTY,

Cambridge, Massachusetts, December, 1903.

\title{
ON THE THEOREM OF ANALYSIS SITUS RELAT- ING TO THE DIVISION OF THE PLANE OR OF SPACE BY A CLOSED CURVE OR SURFACE.
}

\author{
BY MR. L. D. AMES.
}

(Read before the American Mathematical Society, December 29, 1903.)

\section{The Theorem for the Plane.}

JORDAN has proved $*$ that a simple $\dagger$ closed plane curve divides the totality of the points of the plane not on the curve into an interior and an exterior region. He assumes the theorem for the polygon. For the regular curve $\dagger$ we here give a simple proof which goes back to fundamental principles, includes the polygon as a special case, and can be extended to the analogous theorems in more than two dimensions.

In two dimensions the method is as follows: Let a point be conceived as a number pair, the plane as the totality of such points, and a curve as an assemblage of points defined by one or more equations. The fundamental conception on which our proof rests is that of the order of a point. The order is a point function, uniquely defined for every point of the plane not lying on the curve, and its value is always an integer, positive, negative or zero. Before proceeding to this definition we will first define the angle $\theta$ from the line

\footnotetext{
* Jordan, Cours d'Analyse, 2d ed. 1893, vol. 1, 8, 96-102.

† A simple curve shall be defined as a continuous curve without multiple points, and a regular curve as a continuous curve such that the portion join ng any two points of the curve consists of a finite number of pieces, each of which is simple and has a continuous tangent at all its points, inclusive of its extremities. Similarly for surfaces.
} 
to the line

$$
l: y=a_{2} t+b_{2}, \quad x=a_{1} t+b_{1}, \quad\left(t_{0} \leqq t \leqq t_{1}\right),
$$

$$
l^{\prime}: \quad x=a_{1}^{\prime} t+b_{1}^{\prime}, \quad y=a_{2}^{\prime} t+b_{2}^{\prime}, \quad\left(t_{0}^{\prime} \leqq t \leqq t_{1}^{\prime}\right),
$$

as any simultaneous solution of the equations

$$
\sin \theta=\kappa\left|\begin{array}{ll}
a_{1} & a_{2} \\
a_{1}^{\prime} & a_{2}^{\prime}
\end{array}\right|, \quad \cos \theta=\kappa\left|\begin{array}{rr}
a_{2} & -a_{1} \\
a_{1}^{\prime} & a_{2}^{\prime}
\end{array}\right|,
$$

where $\kappa$ is the positive number $\kappa=\left(\sqrt{a_{1}^{2}+a_{2}^{2}} \cdot \sqrt{{a_{1}^{\prime 2}+a_{2}^{\prime 2}}^{2}}\right)^{-1}$.

Let a closed curve be defined by the equations

$$
x=\phi(t), \quad y=\psi(t),
$$

where $\phi$ and $\psi$ are continuous functions of $t$ having the primitive period $\omega$,

$$
\phi(t+\omega)=\phi(t), \quad \psi(t+\omega)=\psi(t) .
$$

Let $O$ be any fixed point not on the curve, and let $P_{0}$ and $P$ be a fixed and a variable point respectively, both on the curve. Let $\theta(t)$ be such a determination of the angle $P_{0} O P$ that it is a continuous function of the parameter $t$ of the point $P$. Then

$$
\theta(t+\omega)=\theta(t)+2 n \pi,
$$

where $n$ is a positive or a negative integer, or zero. This number $n I$ define as the ORDER of the point $O$ with respect to the curve. The absolute value of $n$ is independent of the position of $P_{0}$, or of any consistent choice of the parameter. The sign of $n$ depends only on the choice of the parameter $t$. This choice corresponds arithmetically to the geometric sense in which the curve is described. The points of the order of a given point can be shown to form one or more continua.

THEOREM. A simple regular closed plane curve divides the totality of the points of the plane not on the curve into two continua, of each of which the curve is the total boundary.

The proof is given by the aid of two lemmas. The first asserts that, in any region about a simple point of a regular closed plane curve (with or without multiple points), there are two points of two orders differing by unity, and it is shown that a continuous curve joining them must cut the given curve. From this it follows that the points of the plane not on the given curve form at least two continua. 
The second lemma asserts that, if a simple regular curve (with the possible exception of one or both of its extremities, in case it is not a closed curve) lies within a region $R$, then the totality, $R^{-}$, of points of $R$ not on the curve forms at most two continua ; if at least one end point of the curve is interior to $R$, the points of $R^{-}$form one continuum. This is proved first for a curve which can be represented by the equation

$$
y=\phi(x), \quad\left(x_{0} \leqq x \leqq x_{1}\right),
$$

where $\phi(x)$ and $\phi^{\prime}(x)$ are single-valued and continuous, by showing that any point of $R^{-}$can be joined to one of two preassigned points in $R^{-}$by a continuous curve in $R^{-}$. This is done by the aid of a neighborhood of an arc of the given curve, which is divided by the curve into two continua. If an end point of the curve is interior to $R$, these can be joined into one continuum. If the whole regular curve lies in a finite region, it can be divided into a finite number of such parts, which are thought of as constructed successively, and the lemma is shown to be true at each stage. If the curve is infinite, it is sufficient to consider three arbitrary points in $R^{-}$and a suitable finite portion of the curve. Thus the lemma is extended to the whole curve. If, in particular, $R$ be taken as the whole plane, it follows that the points of the plane not on a given regular simple curve form at most two continua. It is then shown that every point of the curve is a boundary point of each region, and that every boundary point of either region is a point of the curve. This proves the theorem.

The order of an exterior point is 0 , that of an interior point is \pm 1 . Hence we have obtained an arithmetic proof of the following theorem, which we state for greater clearness in geometric language: When $P$ describes the simple regular closed curve $C$, the angle $\theta$ comes back to its initial value when $O$ is an exterior point of $C$, and increases by $2 \pi$ or $-2 \pi$ when $O$ is an interior point.

\section{The Corresponding Theorem for Surfaces.}

Consider any finite surface $R$ in space of three dimensions. Let $R^{\prime}$ be a region of the surface whose boundary $C^{\prime}$ is a single simple closed curve which divides the surface into two or more regions. Define the positive sense of this boundary arbitrarily. Let $R^{\prime \prime}$ be any other such region whose boundary 
is $C^{\prime \prime}$. Draw curves which, together with $C^{\prime}$ and $C^{\prime \prime}$, divide $R$ into mutually exclusive regions $R_{i}(i=1,2,3, \ldots)$ each bounded by only one simple closed curve $C_{i}$, where $C_{i}$ divides $R$ into two or more regions. If it is then possible to define the positive sense for each of these boundaries so that, when each boundary is traced in the positive sense, each segment common to two of the curves $C_{i}$ will have been traced once in one direction and once in the other, the surface is said to be bilateral.* The present discussion is confined to bilateral surfaces. When the positive sense of the boundaries of the regions $R_{i}$ is thus defined, the positive sense of the boundary of $R^{\prime \prime}$ is defined to be the same as the positive sense of the boundaries of the region or regions $R_{i}$ of which the boundary of $R^{\prime \prime}$ consists, in so far as these boundaries coincide. Thus the positive sense of any simple closed boundary of a region on the surface is defined, and by means of this definition the positive normal is defined wherever it exists.

Let $O$ be any point not on the surface. Refer the surface to a system of spherical coördinates with origin at $O$. Let $R$ be any region on the surface, and let its boundary be the curve $C$ whose equation is $\phi=f(\theta)$. If $R$ does not have points on both the positive and negative polar axes $\phi=0, r>0$, and $\phi=\pi, r>0$, define the solid angle $\Theta(O, R)$ subtended by $R$ at $O$ as follows:

$$
\Theta(O, R)=\int_{C}(\cos \phi \pm 1) d \theta
$$

where the upper sign is used if $R$ and $C$ have no point on the positive polar axis $\phi=0, r>0$, and where the lower sign is used if $R$ and $C$ have no point on the negative polar axis $\phi=\pi$, $r>0$. Both signs yield the same value when $R$ has no point on the polar axis $\phi=0$. Any region $R$ can be divided into parts $R_{i}$, each falling into one or both of these classes, and $\Theta(O, R)$ is then defined as $\Sigma \Theta\left(O, R_{i}\right)$. The solid angle is thus uniquely and consistently defined. The order of a point in two dimensions is a limiting case as the point $O$ approaches the plane. The solid angle is susceptible of an algebraic sign, and is continuous for all positions of $O$ not on the surface. The spherical excess of a region on the surface of a sphere, and of the corresponding solid angle, is defined as the limit of the spheri-

* Cf. Möbius, Gesammelte Werke, vol. 2, p. 477. 
cal excess of the inscribed polygon. By subdividing the region by meridians and parallels, and allowing the subdivisions to approach zero, the solid angle is shown to equal the spherical excess.

If $R$ is a closed surface,

$$
\Theta(O, R)=4 n \pi
$$

where $n$ is a positive or negative integer, or zero. The number $n$ is defined as the ORDER of the point $O$ with respect to the surface. The order of $O$ with respect to a closed surface is equal to the order of $O$ with respect to a plane section through $O$, when this is defined. The remainder of the proof that a simple regular closed surface divides space into two continua, of each of which the surface is the total boundary, is similar to that in two dimensions. The method here used is equally applicable to space of $n$ dimensions.

UNIVERsity OF Missouri, October, 1903.

\section{THE THEORY OF WAVES.}

Leçons sur la Propagation des Ondes et les Equations de l'Hydrodynamique. Par Jacques Hadamard. Paris, A. Hermann et Fils, 1903 . xiii +375 pp.

Is handling mathematics and especially mathematical physics, in which the data are never quite so extramundane as in some branches of pure mathematics, there are two things which serve to determine the correctness of results. One is intuition ; the other, rigorous accuracy in analysis. The great investigator like Gibbs, even when dealing with such critical questions as arise in statistical mechanics, never has need of epsilons and deltas to insure the results against error. There are others of us however to whom the rigorous proof is more convincing and even necessary to conviction. Furthermore, as one proceeds from the more evident to the more refined phenomena of physics, the need of exact demonstrations becomes constantly greater. M. Hadamard has shown us in the Transactions * an example of what may be obtained from the critical rigorous

* Vol. 3 (1902), pp. 401-422. 